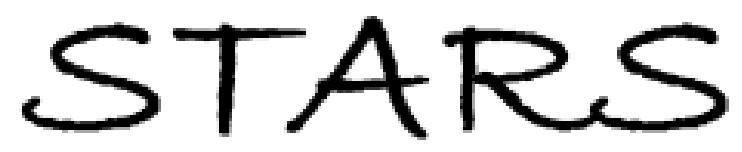

University of Central Florida

STARS

$1-1-2010$

\title{
An empirical potential for silicon under conditions of strong electronic excitation
}

\author{
Lalit Shokeen \\ University of Central Florida \\ Patrick K. Schelling \\ University of Central Florida
}

Find similar works at: https://stars.library.ucf.edu/facultybib2010 University of Central Florida Libraries http://library.ucf.edu

This Article is brought to you for free and open access by the Faculty Bibliography at STARS. It has been accepted for inclusion in Faculty Bibliography 2010 s by an authorized administrator of STARS. For more information, please contact STARS@ucf.edu.

\section{Recommended Citation}

Shokeen, Lalit and Schelling, Patrick K., "An empirical potential for silicon under conditions of strong electronic excitation" (2010). Faculty Bibliography 2010s. 786.

https://stars.library.ucf.edu/facultybib2010/786

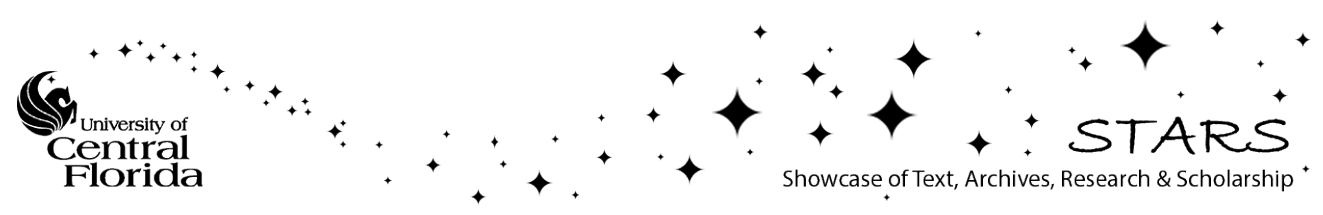




\section{An empirical potential for silicon under conditions of strong electronic excitation}

Cite as: Appl. Phys. Lett. 97, 151907 (2010); https://doi.org/10.1063/1.3499296

Submitted: 13 July 2010 . Accepted: 15 September 2010 . Published Online: 13 October 2010

Lalit Shokeen, and Patrick K. Schelling

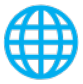

View Online

\section{ARTICLES YOU MAY BE INTERESTED IN}

Thermodynamics and kinetics of silicon under conditions of strong electronic excitation Journal of Applied Physics 109, 073503 (2011); https://doi.org/10.1063/1.3554410

Atomic-scale simulation of the thermodiffusion of hydrogen in palladium

Journal of Applied Physics 114, 063509 (2013); https://doi.org/10.1063/1.4816961

Computational methodology for analysis of the Soret effect in crystals: Application to hydrogen in palladium

Journal of Applied Physics 112, 083516 (2012); https://doi.org/10.1063/1.4758462

\section{Applied Physics Reviews} Now accepting original research 


\title{
An empirical potential for silicon under conditions of strong electronic excitation
}

\author{
Lalit Shokeen and Patrick K. Schelling ${ }^{\text {a) }}$ \\ Department of Physics, Advanced Material Processing and Analysis Center, University of Central Florida, \\ 4000 Central Florida Blvd., Orlando, Florida 32816, USA
}

(Received 13 July 2010; accepted 15 September 2010; published online 13 October 2010)

\begin{abstract}
We present an empirical potential developed for silicon under conditions of strong electronic excitation. We show the essentially athermal nature of the melting transition when the electronic temperature is extremely high. The resulting liquid is shown to be distinct from ordinary liquid silicon. For less intense excitations, we determine the thermal melting temperature and demonstrate the possible existence of a regime where ordinary thermodynamic melting can occur but at a reduced temperature $T_{m}$. We show laser-induced softening of the lattice can lead to lattice cooling for very short time scales ( $\sim 100 \mathrm{fs})$, an effect never before recognized. () 2010 American Institute of Physics. [doi:10.1063/1.3499296]
\end{abstract}

For some time now there has been a great deal of attention given to the processing of materials using laser pulses. Recent advances have enabled the generation of ultrashort (100 fs or less) laser pulses that can generate local carrier densities high enough $\left(\sim 10^{22} \mathrm{~cm}^{-3}\right)$ to cause a destabilization of the lattice. The rapid production of liquid by athermal transitions has been directly observed by several groups, and in situ $\mathrm{x}$-ray diffraction has provided direct visualization of the atomic motion in indium antimonide. ${ }^{1}$ While the experimental evidence for the role of athermal processes is quite strong, there are many unanswered fundamental questions relating to the details of the structural evolution and the relevant time scales. For example, one interesting possibility is that a regime exists where thermal melting transitions occur at a temperature lower than the ordinary melting transition due to softening of the lattice. It might therefore be possible to observe a gradual transition from thermal transitions to purely athermal transitions.

At present, the available theoretical tools are unable to completely resolve these questions. Approaches based on finite-temperature density-functional theory (DFT) (Refs. 2 and 3) and tight-binding ${ }^{4}$ have shown that electronic excitation in silicon leads to melting and a resultant liquid with properties quite distinct from ordinary liquid silicon. Recently it has been shown that electronic excitation in silicon can lead to lattice instabilities as exhibited by transverse acoustic (TA) phonon modes with imaginary frequencies. ${ }^{5}$ However, DFT approaches are limited in their ability to directly model annealing, melting, or ablation. Instead, molecular-dynamics simulation with empirical potentials is often applied to study laser processing and ablation. ${ }^{6}$ However, empirical potentials have not been developed to capture the effect of lattice instabilities due to electronic excitation.

In this letter, we present an empirical potential used to model silicon when the electrons are in a highly excited state. This same basic approach has been applied to metals. ${ }^{7}$ The parameters of the potential are determined by fitting to finite-temperature DFT calculations. This potential is then used to explore the dynamics of silicon after the excitation to very high electronic temperatures $T_{e}$.

${ }^{a)}$ Electronic mail: pschell@mail.ucf.edu.
To provide a database for the empirical potential, we performed finite-temperature DFT calculations using the ABINIT code. ${ }^{8}$ The conditions for our calculations were quite close to that given in Ref. 5. In particular, the local-density approximation was used, with the $3 s, 3 p$, and $3 d$ orbitals included for the valence electrons. The energy cutoff for the plane-wave expansion was taken to be $544 \mathrm{eV}$, and an $8 \times 8 \times 8$ Monkhorst-Pack mesh of k-points was used. Result were obtained for diamond, fcc, bcc, and simple-cubic lattices at seven different volumes each. Calculations were performed for electronic temperatures between $k_{B} T_{e}=0 \mathrm{eV}$ and $k_{B} T_{e}=2.50 \mathrm{eV}$. Starting at $k_{B} T_{e}=0.2 \mathrm{eV}$, the calculations were performed in increments of $0.05 \mathrm{eV}$, thereby generating results for 48 different temperatures. We obtain in each case the cohesive free energy. For the diamond lattice, we obtained results that are in good agreement with prior DFT calculations. ${ }^{2,3,5}$

The empirical potential is based on a recently published modified Tersoff formalism (called the MOD potential here and in Ref. 9), which has been shown to provide an excellent description of the phase behavior and kinetics. ${ }^{9,10}$ For $k_{B} T_{e}$ $=0$, we use the original formulation except with larger cutoffs $\left(R_{1}=3.1 \AA\right.$ and $\left.R_{2}=3.4 \AA\right)$ and $\alpha=1.90$ chosen to give a melting temperature $T_{m}=1688 \mathrm{~K}$ close to experiment. For

TABLE I. Parameters used for the empirical potential for different values of $T_{e}$. The notation follows Ref. 9. Parameters not given in the table are unchanged from the original potential. The constant term $F_{0}$ is the free-energy of an isolated silicon atom at finite $T_{e}$. We also include the phonon frequency at the X-point (in $\mathrm{cm}^{-1}$ ) with the lattice parameter $a=5.429 \AA$ and the DFT value in parenthesis for comparison.

\begin{tabular}{lcccc}
\hline \hline$k_{B} T_{e}(\mathrm{eV})$ & 0.50 & 1.30 & 2.15 & 2.50 \\
$A(\mathrm{eV})$ & 2944.0685 & 2179.9844 & 1827.4731 & 1761.6812 \\
$B(\mathrm{eV})$ & 92.3232 & 53.8369 & 35.7868 & 31.8686 \\
$\lambda_{2}\left(\AA^{-1}\right)$ & 1.223828 & 0.769646 & 0.370933 & 0.243576 \\
$\eta \times \delta$ & 0.610977 & 0.785708 & 0.867687 & 0.906381 \\
$\alpha$ & 1.679913 & 1.3284943 & 1.081435 & 1.027233 \\
$c_{1}$ & 0.214947 & 0.637945 & 1.068782 & 1.155003 \\
$c_{2}$ & 533198.46 & 172192.94 & 164461.57 & 160964.28 \\
$c_{4}$ & 1.090 & 1.234 & 1.387 & 1.450 \\
$F_{0}(\mathrm{eV})$ & 0.0002 & 0.13748 & 0.8928 & 1.3400 \\
$\omega_{\mathrm{TA}}(X)$ & $235(135)$ & $63(69)$ & $-101(-97)$ & $-124(-108)$ \\
\hline \hline
\end{tabular}




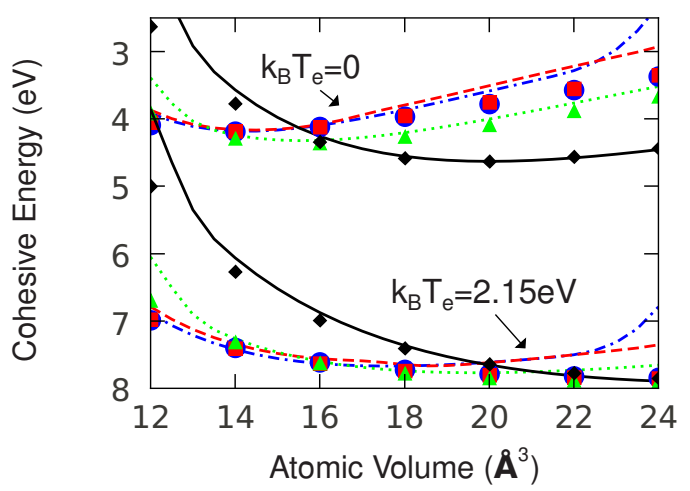

FIG. 1. (Color online) Cohesive free energy per Si atom determined by finite-temperature DFT and the fit empirical potential at two values of $T_{e}$. The DFT results are indicated by symbols (diamonds: diamond; triangles: simple-cubic; squares: bcc; and circles: fcc), and the empirical potential is shown by lines (solid line: diamond; dotted line: simple-cubic; dashed line: bcc; and broken line: fcc).

finite $T_{e}$, the parameters were fit to reproduce the DFT cohesive energies for the fcc, bcc, simple cubic, and diamond structures at several different volumes. Some parameters were found to not be critical, and hence were held fixed. Because the potential for $k_{B} T_{e}=0$ was not fit to the DFT, we fit instead to changes in the free energy curves as a function of electronic temperature $T_{e}$. Also, the DFT energies were shifted to give the experimental value $E_{\text {coh }}=4.63 \mathrm{eV}$ in the equilibrium structure. In Table I, we present the parameters used in the calculations described here. We include in Table I, a constant $F_{0}$ that is added to the empirical potential to take into account the fact that the free energy of an isolated silicon atom will also depend on $T_{e}$. In Fig. 1 we show a comparison of the cohesive free energies predicted by DFT and the empirical potential.

We show the dependence of the $T_{\text {ion }}=0 \mathrm{~K}$ bulk modulus (Fig. 2) and lattice parameter (Fig. 3) on the electronic temperature $T_{e}$. To obtain the lattice parameter and bulk modulus from the DFT calculations, we fit the data to the BirchMurnaghan equation of state. Another important characteristic is the dependence of the phonon frequencies on $T_{e}$. It has previously been shown that the TA branch in diamond silicon is unstable at $k_{B} T_{e}=2.15 \mathrm{eV}$. We give in Table I the TA phonon frequencies $\omega_{\mathrm{TA}}$ at the $X$-point with a comparison to the DFT predictions. In each case, the lattice parameter is fixed at the value $a=5.429 \AA$. While the values of $\omega_{\mathrm{TA}}$ are significantly larger than the DFT results for low $T_{e}$, the over-

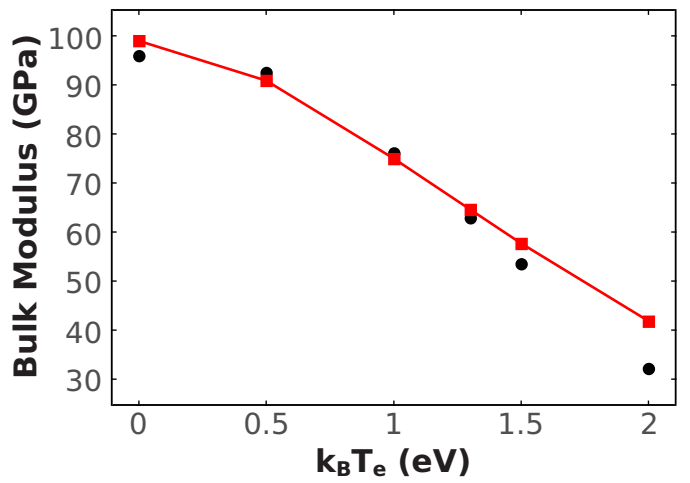

FIG. 2. (Color online) Bulk modulus of the diamond structure computed for the empirical potential (red squares) and DFT (black circles).

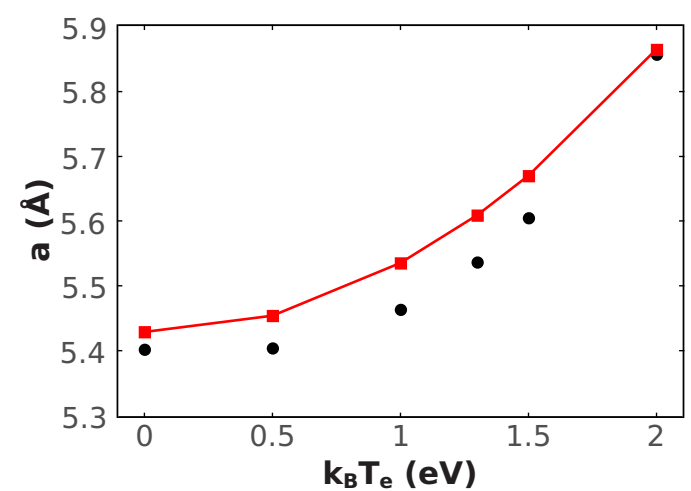

FIG. 3. (Color online) Lattice parameter of the diamond structure computed for the empirical potential (red squares) and DFT (black circles).

all qualitative agreement with DFT is quite good and in fact improves for higher $T_{e}$.

To simulate the effect of an intense laser pulse, we first equilibrate an $8 \times 8 \times 8$ cell with $4096 \mathrm{Si}$ atoms at $T_{\text {ion }}$ $=300 \mathrm{~K}$ with the empirical potential for $k_{B} T_{e}=0$ for 11.05 ps. After this step, we instantaneously change the empirical potential to correspond to strong excitation and continue the simulation at constant energy and volume. We chose constant volume boundary conditions to compare with existing DFT results with the same boundary conditions. ${ }^{2,3}$ As a result of the excitation, the temperature evolves away from $T_{\text {ion }}$ $=300 \mathrm{~K}$. In Fig. 4 we show the evolution of $T_{\text {ion }}$ with time after the excitation. We find that for $k_{B} T_{e}=2.15 \mathrm{eV}$ and $k_{B} T_{e}=2.50 \mathrm{eV}$, the lattice melts and $T_{\text {ion }}$ strongly increases within about $\sim 100 \mathrm{fs}$. We find final temperature $T_{\text {ion }}$ $\approx 580 \mathrm{~K}$ when $k_{B} T_{e}=2.15 \mathrm{eV}$, significantly lower than $T_{\text {ion }} \approx 1700 \mathrm{~K}$ found in previous DFT calculations. ${ }^{2,3}$ We believe that the difference with the DFT results is due to the fact that the starting MOD potential over predicts the energy difference between diamond and the highly coordinated fcc and bcc lattices. Since the fits at finite $T_{e}$ are done to reproduce the changes with $T_{e}$, we have a similar error at all values of $T_{e}$. Hence we predict a smaller change in energy (by less than $0.1 \mathrm{eV}$ per atom) when the system transitions to the highly coordinated liquid, and hence the increase in $T_{\text {ion }}$ is somewhat smaller. However, we find that at $k_{B} T_{e}$ $=2.50 \mathrm{eV}$ that $T_{\text {ion }} \approx 950 \mathrm{~K}$. Interestingly, we find that for $k_{B} T_{e}=1.30 \mathrm{eV}$, the system does not melt and in fact $T_{\text {ion }}$ slightly decreases to $T_{\text {ion }} \approx 230 \mathrm{~K}$. At $k_{B} T_{e}=1.30 \mathrm{eV}$, there is no lattice instability but instead a significant softening of

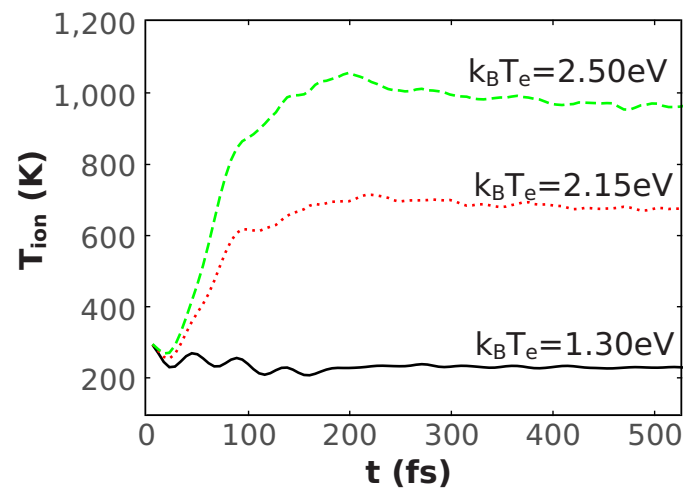

FIG. 4. (Color online) Ionic temperature $T_{\text {ion }}$ plotted as a function of time after electronic excitation to $k_{B} T_{e}=1.30 \mathrm{eV}, k_{B} T_{e}=2.15 \mathrm{eV}$, and $k_{B} T_{e}$ $=2.50 \mathrm{eV}$. 


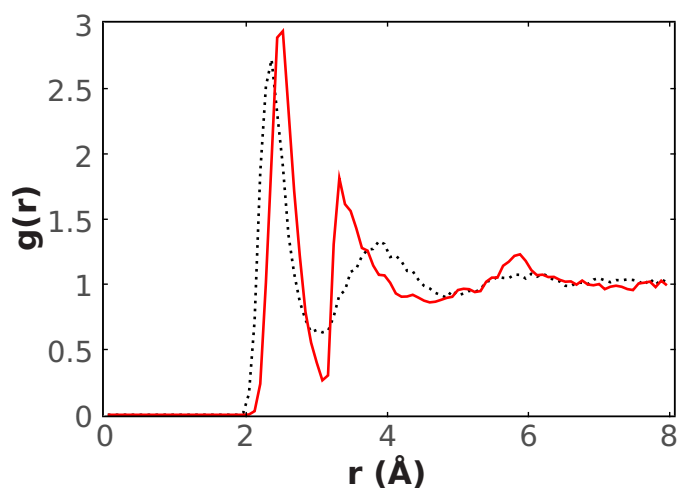

FIG. 5. (Color online) Radial distribution function for melted silicon after excitation at $k_{B} T_{e}=2.15 \mathrm{eV}$ (solid red line). For comparison we show ordinary liquid $\mathrm{Si}\left(k_{B} T_{e}=0\right)$ at $T=1700 \mathrm{~K}$ (dotted black line).

all phonon modes. Hence, the atomic displacements become larger but the average kinetic energy decreases due to weaker restoring forces. However, the excited system is under fairly high stress and we expect the temperature to rise somewhat in a constant pressure simulation.

For the liquid resulting from the excitation, we find a distinct structure compared to ordinary liquid silicon. In Fig. 5 we show the radial distribution function $g(r)$ for the $k_{B} T_{e}$ $=2.15 \mathrm{eV}$ simulation in comparison to a simulation with $k_{B} T_{e}=0$ and $T_{\text {ion }}=1700 \mathrm{~K}$. The first peak in $g(r)$ occurs at $2.55 \AA$ in the excited silicon, compared to $2.42 \AA$ in the ordinary liquid. In contrast to DFT predictions, ${ }^{2,3}$ we see a sharp minimum at $3.13 \AA$. Using this minimum as a cutoff, we find a coordination number of 5.31. However, if we increase the temperature to $T_{\text {ion }}=1700 \mathrm{~K}$, Fig. 6 shows that this minimum largely goes away. In this case, using $4.0 \AA$ as a cutoff, the coordination number is 12.71 , which compares quite well with the DFT value in the range $11-13 .^{2,3}$

Finally we obtain the melting temperature $T_{m}=1278 \mathrm{~K}$ for the $k_{B} T_{e}=0.5 \mathrm{eV}$ case. To determine $T_{m}$, we simulate coexistence of liquid and crystal. ${ }^{10}$ This demonstrates that electronic excitation might lead to a thermal transition at a strongly modified temperature.

In summary, we have developed an empirical potential for silicon that depends on the electronic temperature $T_{e}$. We

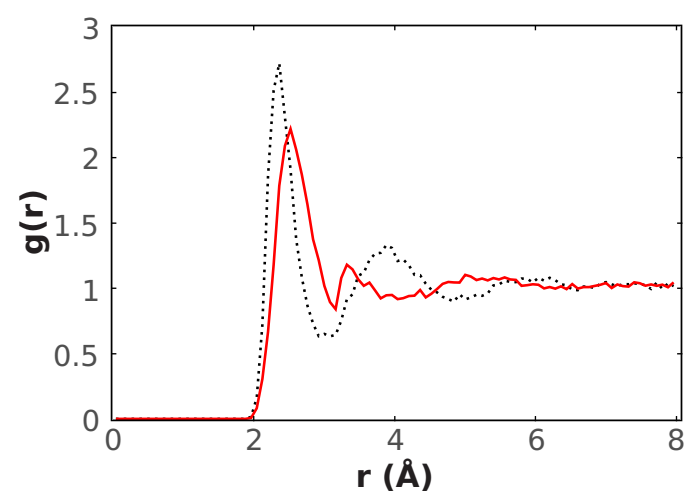

FIG. 6. (Color online) Radial distribution function for $k_{B} T_{e}=2.15 \mathrm{eV}$ (solid red line) and $k_{B} T_{e}=0$ (black dotted line) both computed at $T_{\text {ion }}=1700 \mathrm{~K}$.

have elucidated some aspects of melting in conditions of strong laser excitation. For relatively weak excitation, we find that cooling can occur for short time scales.

This work was supported a grant from the National Science Foundation (Grant No. 0809015).

\footnotetext{
${ }^{1}$ A. Rousse, C. Rischel, S. Fourmaux, I. Uschmann, S. Sebban, G. Grillon, P. Balcou, E. Forster, J. P. Geindre, P. Audebert, J. C. Gauthier, and D. Hullin, Nature (London) 410, 65 (2001)

${ }^{2}$ P. L. Silvestrelli, A. Alavi, M. Parrinello, and D. Frenkel, Phys. Rev. Lett. 77, 3149 (1996).

${ }^{3}$ P. L. Silvestrelli, A. Alavi, M. Parrinello, and D. Frenkel, Phys. Rev. B 56, 3806 (1997).

${ }^{4}$ A. Gambirasio, M. Bernasconi, and L. Colombo, Phys. Rev. B 61, 8233 (2000).

${ }^{5}$ V. Recoules, J. Clerouin, G. Zerah, P. M. Anglade, and S. Mazevet, Phys. Rev. Lett. 96, 055503 (2006).

${ }^{6}$ P. Lorazo, L. J. Lewis, and M. Meunier, Phys. Rev. B 73, 134108 (2006).

${ }^{7}$ S. Khakshouri, D. Alfe, and D. M. Duffy, Phys. Rev. B 78, 224304 (2008).

${ }^{8}$ X. Gonze, J. M. Beuken, R. Caracas, F. Detraux, M. Fuchs, G. M. Rignanese, L. Sindic, M. Verstraete, G. Zerah, F. Jollet, M. Torrent, A. Roy, M. Mikami, P. Ghosez, J. Y. Raty, and D. C. Allan, Comput. Mater. Sci. 25, 478 (2002).

${ }^{9}$ T. Kumagai, S. Izumi, S. Hara, and S. Sakai, Comput. Mater. Sci. 39, 457 (2007).

${ }^{10}$ P. K. Schelling, Comput. Mater. Sci. 44, 274 (2008).
} 